\title{
Therapeutic Drug Monitoring of Anti-TNF Therapy in Inflammatory Bowel Disease
}

Frank I. Scott, M.D., M.S.C.E. and Instructor of Medicine, Division of Gastroenterology, Department of Medicine, University of Pennsylvania, 9 Penn Tower, 1 Civic Center Boulevard, Philadelphia, PA 19104, Phone: 215-554-0623, Fax: 215-615-1688

\section{Gary R. Lichtenstein, M.D.}

Professor of Medicine, Director, IBD Program, Hospital of the University of Pennsylvania, Division of Gastroenterology, Department of Medicine, University of Pennsylvania, 9 Penn Tower, 1 Civic Center Boulevard, Philadelphia, PA 1910

Frank I. Scott: frankis@mail.med.upenn.edu

\begin{abstract}
While anti-TNF agents have had a marked impact in the treatment of inflammatory bowel disease, a significant number of patients lose their response to these medications over time. Clinical trials have demonstrated that antibodies against anti-TNF medications may impact treatment response and increase the risk of infusion reaction. Scheduled dosing and concurrent use of immunomodulators may help to mitigate these risks via inhibiting the formation of these antibodies. The recent availability of assays to measure anti-TNF drug levels and antibodies against anti-TNFs offer the opportunity to assess patients who have lost response with infliximab and adalimumab, and potentially determine the most appropriate therapeutic strategy. There is growing evidence that such testing improves patient outcomes and is cost-effective, although heterogeneity in the assays used in clinical and observational trials has resulted in mixed results.
\end{abstract}

\section{Keywords}

Infliximab; Adalimumab; Certolizumab; Azathioprine; Immunomodulators; Tumor necrosis factor-alpha (TNF-a); Anti-TNF drug levels; Biologic; Antibodies to infliximab (ATIs); Antibodies to adalimumab (ATAs); Crohn's disease; Ulcerative colitis

\section{Introduction}

Anti-tumor necrosis factor-alpha (Anti-TNF) agents have permanently modified the landscape of therapeutic options in inflammatory bowel disease (IBD). These large, monoclonal antibodies against TNF are potentially immunogenic, and several clinical and

\footnotetext{
Conflict of Interest

Frank I. Scott declares that he has no conflict of interest.

Compliance with Ethics Guidelines

Human and Animal Rights and Informed Consent

This article does not contain any studies with human or animal subjects performed by any of the authors.
} 
observational trials have demonstrated that resultant antibody formation against these medications has significant consequences both for drug pharmacokinetics and safety. In this article, we review the available data from clinical and observational trials on the impact of antibodies against anti-TNF agents, the effect of varying serum drug levels of these agents, and the potential clinical applicability of measuring antibodies against anti-TNFs and drug levels in those that have lost response.

Crohn's disease (CD) and ulcerative colitis (UC), the two main subtypes of inflammatory bowel disease (IBD), have been demonstrated to have an overly active immune response to the gut enteric flora, culminating in bowel inflammation and ulceration. Patients with IBD often experience abdominal pain and diarrhea, and are at an increased risk for malabsorptive syndromes, intestinal resection or colectomy, and colorectal cancer. On a societal level, these diseases are responsible for a significant health and economic burden.

Traditional IBD therapies have focused on modulation of the inflammatory response using topical therapies such as 5-aminosalicylates and immunomodulators such as azathioprine, 6mercaptopurine, and more recently, methotrexate. Despite these agents' utility, the majority of patients with moderate to severe disease require further therapy.

The development of a class of medications targeting tumor necrosis factor- $a$ (TNFa), a key cytokine involved in the aggressive immune response in IBD, has yielded new therapeutic options for patients with moderate to severe CD and UC. Infliximab, a chimeric IgG molecule consisting of a human $\mathrm{Fab}^{\prime}$ fragment combined with a murine Fc fragment, was the first of these agents to be assessed in IBD, demonstrating clinical efficacy in inducing and maintaining remission in both $\mathrm{CD}$ and UC. Infliximab has been shown to be beneficial in the management of fistulizing disease and appears to be effective in early studies in preventing post-operative recurrence. Adalimumab, a completely humanized IgG molecule, has similarly been approved for use in both CD and UC, demonstrating efficacy in treating both of these diseases. One advantage of this agent is its subcutaneous mode of delivery, whereas infliximab is delivered via IV infusion. A third agent, certolizumab pegol, which is a pegylated human $\mathrm{Fab}^{\prime}$ fragment, is only approved for use in $\mathrm{CD}$. It is currently being assessed for efficacy in UC.

The availability of these agents has resulted in a major paradigm shift in the management of moderate to severe IBD. The previous therapeutic standard of increasing the level of immunosuppression with disease progression is being supplanted by more aggressive early therapy with a combination of both a biologic agent and an immunomodulator in an effort to modify the overall course of IBD, particularly in those patients who have characteristics of aggressive disease. Several studies have shown that patients have higher rates of remission and response when using biologic agents compared to immunomodulators, and that the combination of these agents provides even greater benefit ${ }^{1-3}$.

\section{Loss of response and antibodies to anti-TNF agents}

While the advent of biologic therapy has brought significant benefit to a large percentage of patients with IBD, there remain a significant proportion of patients that lose response over time (termed secondary loss of response). This has been demonstrated in the clinical trials of 
maintenance therapy for infliximab, adalimumab, certolizumab, and combination therapy ${ }^{4-6}$. The underlying etiology for loss of response to anti-TNF agents is incompletely understood. Two primary mechanisms that have been hypothesized in the case of infliximab are the formation of antibodies to infliximab (ATIs), and fluctuations in circulating drug levels brought about by altered drug clearance. These mechanisms are also thought to play a significant role in the loss of response for other anti-TNF agents as well.

Antibodies to infliximab (ATIs) have been a recognized entity since the initial use of this agent in humans. The structure of the monoclonal anti-TNF antibodies lends to their immunogenicity. Infliximab, adalimumab, and certolizumab are large antibodies or antibody fragments, and can be readily processed via phagocytosis to stimulate antibody formation ${ }^{7,8}$. As previously noted, infliximab is a chimeric antibody consisting of both murine and human components. It was initially felt that the murine component of this molecule contributed significantly to its immunogenicity. Adalimumab was designed in an attempt to reduce immunogenicity by completely humanizing the antibody. It is not surprising that this large molecule has been shown to stimulate antibody formation in clinical trials as well ${ }^{4,9,10}$. Certolizumab has demonstrated the ability to stimulate antibody formation in spite of its pegylated formulation, with $8 \%$ of patients receiving the drug developing antibodies to certolizumab (ATCs) in the PRECISE 1 trial ${ }^{11}$. This rate of antibody formation increased to $17.1 \%$ in longitudinal use if there was any interruption in scheduled dosing ${ }^{6}$.

Antibodies formed against infliximab and antibodies to adalimumab (ATAs) can be of any of the immunoglobulin subtypes, and can bind several sites on the monoclonal antibody, including the TNF binding site of the molecule, thus inactivating the molecule. There are several potential epitopes for antibody formation, allowing for antibodies directed at different components of the TNF molecule ${ }^{12}$. The Fab fragment appears to be the favored site compared to the Fc region. Regardless of binding site, ATI-infliximab complexes are inherently larger than infliximab alone. Even without direct inactivation, ATIs appear to increase the clearance rate of infliximab ${ }^{13,14}$. It is important to note, however, that early pharmacokinetic studies have typically been conducted using ELISA-based assays to measure fluctuations in drug levels. These assays may not fully describe the pharmacokinetics of infliximab in the presence of ATIs, and further research is required ${ }^{13,14}$.

There have been several recent advances in the methods used to measure anti-TNF antibodies in patients receiving these agents (Table 1). The most commonly used assay to measure ATIs is the solid-phase, or sandwich, enzyme-linked immunosorbent assay (ELISA $)^{7}$. In this assay, the patient's serum is added to wells on a plate that have been coated with infliximab. Labeled infliximab is then added to these wells after washing, which binds to another branch of the ATI molecule, allowing for detection the antibodies ${ }^{7}$. While this assay is commonly utilized due to its simplicity, it has several known shortcomings, including false positives due to binding of other serum immunoglobulins not specific to antiTNF and sensitivity to levels of infliximab in the blood ${ }^{8,15}$.

The fluid-phase radioimmunoassay, or RIA, has several advantages over the solid-phase ELISA assay. To perform the RIA, serum samples are incubated with soluble, labeled 
antigen. An additional anti-Fc antibody is then added, and the complex of ATI-radiolabelled antigen and anti-Fc is precipitated out in centrifugation for measurement ${ }^{16}$. This assay is not inhibited by the presence of infliximab in the serum, and can measure ATIs in its presence. Furthermore, it can also measure free infliximab levels in the presence of ATIs ${ }^{17}$. The major drawback of this assay is the use of radiolabelled markers ${ }^{8}$.

Another recently developed assay employs high-pressure liquid chromatography (HPLC), and is called the HPLC-mobility shift assay (HMSA). This liquid-phase assay has the advantage of an additional dissolution phase that can free drugs from antibodies. After this step, serum samples are mixed with fluorescent-labeled infliximab to measure antibodies, or fluorescent-labeled TNF to measure infliximab. Concentrations are measured via HPLC. ${ }^{18}$ This assay is not sensitive to interference by other antibodies, possesses increased sensitivity compared to ELISA, and can measure all subtypes of immunoglobulins to infliximab. It also does not require the radioactive component used in RIA.

An additional new assay to detect anti-TNF drug levels and antibodies was recently developed using electrochemiluminescence as well. This solid-phase assay is capable of determining both drug levels and ATIs, and can measure the presence of ATIs in the presence of infliximab. The presence of ATIs does interfere with the ability of this assay to measure drug levels accurately, however, and this new assay has not yet been validated in comparison to the RIA, HMSA, or ELISA assays. ${ }^{19}$

Factors associated with ATI formation-Several studies have assessed the incidence and impact of ATIs in clinical care (Table 2). Two key factors that appear to increase the incidence of ATIs are dosing frequency and concomitant use of immunosuppression. With respect to dosing frequency, there appears to be a clear correlation between episodic dosing and the development of ATIs. Two studies assessing the efficacy of infliximab in refractory and fistulizing $\mathrm{CD}$ using initial loading doses followed by episodic therapy demonstrated ATI rates from 36 to $61 \%$ and between 42 and $75 \%$, respectively, among those not receiving additional immunosuppression ${ }^{20,21}$. Similarly, Vermeire and colleagues appreciated an ATI incidence of $73 \%$ in a cohort of individuals with refractory and fistulizing CD receiving episodic therapy; the ATI rate decreased to 44-48 \% when concomitant immunomodulators were used ${ }^{22}$. These rates are much higher than those seen in trials using scheduled dosing of infliximab. In the ACCENT 1 trial, all patients received an initial induction dose of infliximab, and responders were randomized to maintenance therapy or placebo. ATI rates among those receiving scheduled dosing ranged between 6-10 $\%$ in this study and subsequent subgroup analysis ${ }^{5,23}$. ATI rates were higher in those randomized to placebo after induction, ranging from $28-30 \%$. Rates were lower among those receiving immunosuppression compared to those who did not. Similar results were appreciated by Maser and colleagues, who compared the efficacy of episodic to scheduled dosing in a prospective cohort with a median of 88 weeks of follow-up ${ }^{24}$. In this study, $21 \%$ of patients developed ATIs; when stratified by dosing schedule, those receiving episodic therapy had an ATI incidence of $39 \%$ compared to $16 \%$ in those who received regularly scheduled dosing $(\mathrm{p}<0.05)$. Similarly lower rates of ATIs were appreciated in the ACT I and ACT II trials of moderate to severe UC, which utilized induction dosing followed by 
scheduled maintenance therapy, with ATI rates of 6.1 to $6.4 \%{ }^{25}$. Based on the data from these trials, it is clear that episodic dosing increases the risk of ATI formation.

Another factor that impacts the incidence of antibodies is concomitant immunosuppressant use such as 6-mercaptopurine, azathioprine, or methotrexate. Baert et al. appreciated an ATI formation in $75 \%$ of those without concomitant immunosuppression, compared to $43 \%$ in those taking azathioprine, $6 \mathrm{MP}$, or methotrexate $(\mathrm{p}<0.001)^{20}$. Farrell et al. also appreciated this difference, with $63 \%$ of those not receiving immunosuppressants developing ATIs compared to $24 \%$ on additional agents $(\mathrm{p}=0.007)^{21}$. Vermeire et al. appreciated an ATI incidence of $73 \%$ in those on infliximab monotherapy, compared to $42-48 \%$ on combination therapy ${ }^{22}$. Similar results have been appreciated in scheduled therapy. Colombel noted ATI formation in $14.6 \%$ of patients using infliximab monotherapy compared to $0.9 \%$ of those receiving combination therapy in the SONIC trial, which assessed the efficacy of combination therapy compared to monotherapy with either infliximab or azathioprine $)^{1}$. Decreased ATIs were also appreciated in the ACCENT I trial ${ }^{5}$. Similar results were reported by Van Assche, and have been seen in UC trials ${ }^{25,26}$.

Combination therapy with immunomodulators has been shown to inhibit anti-TNF antibody formation in clinical trials for both adalimumab and certolizumab (TABLE 2). In the Clinical Assessment of Adalimumab Safety and Efficacy Studied as Induction Therapy in Crohn's Disease trial, or CLASSIC II trial of maintenance adalimumab, $3.8 \%$ of patients not on additional immunosuppressants developed ATAs, compared to none of those who did receive concomitant immunosuppressants ${ }^{4}$. Similar results were appreciated in the Ulcerative Colitis Long-Term Remission and maintenance with Adalimumab 2, or ULTRA2, trial, with $2.9 \%$ of patients developing ATAs, all of who were on monotherapy ${ }^{27}$. Similarly, in a clinical trial of maintenance therapy with certolizumab pegol in Crohn's Disease, $12 \%$ of patients on monotherapy developed ATCs compared to $2 \%$ who were receiving combination therapy ${ }^{6}$.

Clinical impact of antibodies to anti-TNF agents-The clinical impact of ATIs in clinical trials has been inconsistent. In the episodic treatment study by Baert et al., ATI concentrations $>8 \mu \mathrm{g} / \mathrm{mL}$ predicted a shorter duration of response compared to those who had lower levels of ATIs ${ }^{20}$. This was also reported by Farrell et al., where those with ATIs had shorter durations of response (28 days) compared to those without ATIs (61 days, $\mathrm{P}=$ $0.007)$ and lower response rates $(14 \% \text { vs. } 52 \%, \mathrm{p}=0.0005)^{21}$. Vermeire et al. also appreciated shorter treatment duration in their episodic dosing trial, though the difference in duration was not statistically significant ${ }^{22}$.

Similar results have not, however, been seen in trials of scheduled dosing,. In a subgroup analysis of the ACCENT I trial, Hanauer and colleagues did not see an association between the presence of ATIs and outcomes ${ }^{23}$. Similarly, Maser et al. did not find this association in their cohort study ${ }^{24}$. The SONIC trial did appreciate improved remission rates at weeks 26 and 50 among those who were ATI inconclusive ${ }^{1}$. As this study used ELISA-based methods to detect antibodies, the authors hypothesize that indeterminate results signified continued circulating infliximab, and that this explained the improved response in this subset of patients. In summary, in initial studies of episodic dosing in $\mathrm{CD}$, there appears to be a 
reduced duration of response to infliximab with ATIs. With scheduled therapy, however, there are several studies that did not demonstrate this relationship, although the SONIC trial did note improved responses in those with an indeterminate ELISA.

The impact of antibodies to anti-TNFs in UC is less clear. As opposed to the results seen for $\mathrm{CD}$, the ACT trials of scheduled therapy with infliximab for induction in UC appreciated an increased rate of clinical response in those subjects that were ATI-positive or inconclusive, compared to those that were ATI-negative. There was a very high rate of inconclusive ELISA results in this study, however. The authors hypothesized that undetectable infliximab was the reason why patients with negative ATI have a poorer response ${ }^{25}$. The picture was further complicated by the results of a prospective trial of scheduled dosing of infliximab in UC carried out by Seow and colleagues. In this study, there was no significant impact of ATI status on clinical response. They did, however, see improved rates of remission, endoscopic findings, and lower rates of colectomy in those with an ATI-inconclusive result ${ }^{15}$.

The clinical impact of ATAs is also an area of active research. Karmiris and colleagues examined the incidence and impact of ATAs in a cohort of 168 individuals through induction and maintenance therapy ${ }^{28}$. In 130 patients who had serum samples available for assessment, $9.2 \%$ were positive for ATAs. No correlation was appreciated between ATAs and induction or maintenance of remission. However, in a much smaller cross-sectional study of 40 patients with CD treated with ADA in Japan, using an HMSA assay and novel ELISA to detect ATAs, $35 \%$ of patients were found to have these antibodies ${ }^{9}$. Patients with ATAs had significantly higher CDAIs, ESR, and CRP levels compared to those who did not. Studies such as this highlight the need for further prospective research using these newer antibody detection techniques to help delineate this association.

The impact of antibodies to certolizumab (ATCs) was assessed in two clinical trials. In the PRECISE -2 trial, ATCs had no impact on clinical outcomes during the induction phase, and in the maintenance phase there was a non-significant reduction in those who maintained remission through week 26 (71\% versus $62 \%$ ), though the overall number of patients who were antibody-positive was low 6 . Based on the sample size, it is possible that this study was not appropriately powered to detect a difference adequately. Similarly, in the WELCOME trial, where approximately $6 \%$ of patients developed ATCs, there was no association between antibody formation and clinical outcomes ${ }^{29}$.

In summary, there appears to be a correlation between drug efficacy and ATIs in the setting of episodic dosing, though the impact of ATIs appears to be negligible when scheduled dosing is used. For ATAs, clinical trials did not appreciate an impact on efficacy, though the more recent application of HMSA assays has suggested there may be a deleterious effect. Lastly, ATCs did not appear to have a significant impact in two clinical trials using conventional ELISAs.

\section{The impact of anti-TNF antibodies on drug safety}

ATIs are also thought to be associated with an increased risk of adverse events during infusion. Typical infusion reactions that can occur while receiving infliximab include fever, 
chills, rigors, nausea and vomiting, dyspnea, and headache. Several studies have noted a relationship between ATI seropositivity and the risk of infusion reactions. Baert et al. detected this association, and this risk was increased with higher ATI concentrations. The relative risk of infusion reaction was 2.4 for those with ATI $>8 \mu \mathrm{g} / \mathrm{mL}^{20}$. In a cohort studied by Farrell et al., all seven patients with infusion reactions were ATI positive. Unlike treatment response, this increased risk of infusion reaction was also appreciated with scheduled therapy. In the ACCENT-1 trial, $38 \%$ of patients who were ATI-positive had infusion reactions, compared to $24 \%$ who were ATI-negative. Similar results were noted in a prospective study by Maser and colleagues ${ }^{23,24}$. A similar relationship has been found in several trials in UC, including the ACT 1 and 2 trials, as well as in the prospective cohort study by Seow and colleagues ${ }^{15,25}$.

\section{Impact of Anti-TNF drug levels on clinical response}

As with any pharmacologic agent, anti-TNF levels fluctuate with dosing, with potential peaks and troughs as the body metabolizes the drug via various means. While the clearance of large monoclonal antibodies is an area of active research, it appears to involve several mechanisms, including endocytosis with proteolytic catabolism in the reticuloendothelial system (RES). This process becomes more efficient when monoclonal antibodies are crosslinked to other host-derived antibodies. ${ }^{30}$ Another mechanism of degradation is internalization after the binding of cell wall-bound antigens such as neonatal Fc receptors, which are found on the vascular endothelial cells within the RES. After binding these cellbound antigens, the antibody is phagocytosed with subsequent lysosomal degradation ${ }^{30}$. These mechanisms are thought to be enhanced by antibodies to anti-TNFs.

As the concentration of monoclonal antibodies in the serum decreases over time, there is less available drug to bind TNF-a, potentially leading to decreased efficacy. The plasma concentrations of anti-TNFs correlate in a linear fashion with the dosage given, though the half-life differs between agents. The median half-life of infliximab is thought to between 7.7 and 9.5 days, whereas the median half-life of the subcutaneously injectable agents adalimumab and certolizumab is longer, at approximately 2 weeks 8 .

Numerous efforts have been made to quantify the effect of anti-TNF levels on drug efficacy. These attempts have been hampered by aspects of study and assay design. There have been differences in dosing of the three main anti-TNFs and in dose scheduling for infliximab in trials, not to mention the potential effects of dose modification and escalation at the individual patient level. Widespread utilization of the ELISA assay has also been problematic; despite ease of use, the inability to adequately measure drug levels in the setting of ATIs has significantly hampered infliximab level measurement, both in clinical trials and in clinical practice. Furthermore, the selection of clinically-oriented, subjective outcomes such as the CDAI in larger clinical trials has also complicated measuring an association due to potential bias introduced by the scoring system itself. Therefore, while many trials have reported data on drug levels, and in particular, infliximab trough levels, this data must be interpreted with caution. Furthermore, the clinical impact of dose and schedule modulation in response to these levels has only recently been assessed, and requires further research for all three of the available agents on the market for IBD. 
Several studies have examined the association between infliximab levels and clinical response, both in episodic and scheduled dosing. Baert et al. found that infliximab levels 4 weeks after an infusion that were greater than $12 \mu \mathrm{g} / \mathrm{mL}$ were associated with a median duration of response of 81.5 days compared to 68.5 days in those with levels lower than 12 $\mu \mathrm{g} / \mathrm{mL}^{20}$. In addition, concurrent immunosuppression was correlated with higher drug levels. Maser et al. appreciated a similar association, also noting reduced endoscopic scores and lower CRP levels with higher infliximab concentrations ${ }^{24}$. Similar associations have been appreciated in scheduled dosing studies as well. In a randomized, open-label study of immunomodulator discontinuation conducted by Van Assche and colleagues, infliximab levels were again inversely correlated with CDAI and CRP levels. Similarly, in the SONIC trial, $72.8 \%$ (110 of 151) patients receiving infliximab either alone or in combination with azathioprine who had infliximab trough levels $>1.0 \mu \mathrm{g} / \mathrm{mL}$ were in remission at week 30 , compared to $58.2 \%$ (32 of 55) of those who had lower infliximab levels ${ }^{1}$. Similar results have been appreciated in UC by Seow and colleagues. The remission rate was $69 \%$ in those with detectable serum concentrations of infliximab in this prospective cohort study, compared to a remission rate of $15 \%$ in those who had undetectable levels ${ }^{15}$. Detectable infliximab levels were also associated with increased mucosal healing in a multivariate logistic regression model (OR 7.3, 95 \% CI 2.9-18.4), and undetectable levels were associated with increased risk of colectomy (OR 9.3, $95 \%$ CI 2.9-29.9). In an additional subgroup analysis of data from ACT 1 and ACT 2 by Reinisch and colleagues, infliximab levels were higher in those achieving clinical remission, mucosal healing, and clinical response than in those who were not ${ }^{25,31}$.

Adalimumab levels have also been assessed for correlation with clinical outcomes. A recent post hoc analysis of both the CLASSIC I and CLASSIC II trials of serum adalimumab levels assessed 258 patients who had stored serum samples available. Their regression analysis demonstrated a small, but significant correlation between adalimumab levels and clinical remission at week 4, though this relationship was not appreciated at later time points in the study $^{32}$. In a larger trial of 130 patients receiving maintenance therapy with adalimumab who had available serum samples, there was no relationship appreciated between outcomes at 4 weeks and serum trough levels of adalimumab, though levels were significantly lower in those who discontinued adalimumab use $(\mathrm{p}=0.012)^{28}$. Drastich examined the impact of adalimumab levels in a subgroup of 48 patients receiving adalimumab, of whom 24 were in both clinical and endoscopic remission. Twenty-one of 24 patients meeting these criteria had adalimumab levels $>2 \mu \mathrm{g} / \mathrm{ml}$. However, in this study, 17 of 24 who did not reach deep remission also had adalimumab levels above this cutpoint ( $\mathrm{p}=\mathrm{N}$.S.). Therefore, the impact of drug levels in adalimumab appears to be less clear-cut than that seen with infliximab, warranting further research.

As with adalimumab, the impact of certolizumab levels on drug efficacy in CD also remains unclear. In the PRECISE-4 trial, a group of individuals who initially responded to certolizumab, but then lost response by week 26 in the original PreCISE- 2 trial were given the opportunity to enroll in an open-label extension with a single extra dose of certolizumab $400 \mathrm{mg}$ subcutaneously. A total of $63 \%$ of patients had a response after receiving this additional dose. However, when examining mean plasma concentrations between those who responded and those who did not, there was no significant difference between groups ${ }^{33}$. In 
another trial of certolizumab pegol in patients who had become intolerant to or lost response to infliximab, subjects received open-label induction at weeks 0,2 , and 4 , with responders then being randomized to two different certolizumab dosing regimens ( $200 \mathrm{mg}$ every 2 weeks vs. every 4 weeks). No significant difference was observed between these two arms of the trial, and serum concentrations of certolizumab were not correlated with response ${ }^{29}$. However, in a subgroup of patients with active disease after 6 weeks of induction who entered an open-label phase of the trial, there was an association between subsequent remission and the two higher quintiles of certolizumab levels ${ }^{34}$. Further research is needed to assess the impact of certolizumab levels given these conflicting results.

\section{Utilizing anti-TNF drug levels and antibodies in clinical practice}

Given the potential correlation between ATIs and infliximab drug levels with clinical response and loss of response, there has been a growing body of literature assessing how measurements of both antibodies and drug levels may impact clinical decision-making. As noted, there is some evidence to suggest that infliximab drug concentrations are correlated with clinical response, and also evidence that there is variation in these concentrations in different individuals ${ }^{8}$. Several algorithms have been presented on how to interpret the information derived from these laboratories. Most authors agree on the following (Figure 1): If ATIs are present, one should change to an alternative anti-TNF. However, if ATIs are low or undetectable, serum trough levels are then used-if low, the drug dose should be increased or dosing frequency decreased. If there are adequate drug levels, then one should consider an agent with an alternative mechanism of action ${ }^{16,35}$. Some also advocate for the use of endoscopy for assessment of disease activity in those who have negative ATIs and a therapeutic IFX level before switching to an alternative agent as well ${ }^{16}$.

Several studies have attempted to assess the impact of such algorithms in clinical practice. A recent retrospective study by Afif and colleagues examined the impact of therapeutic drug monitoring in a cohort of 155 patients with IBD in a retrospective review, looking at the impact of the laboratory data on the treatment decisions and outcomes. Seventy-one percent of patients had had a loss of response or only partial response, prompting ATI and infliximab trough testing. Thirty-five patients were ATI positive; 12 of these patients switched to another anti-TNF, and of these, $11(92 \%)$ had a response. Six patients underwent dosing escalation, with only one having an improvement in symptoms (17\%, $\mathrm{p}<0.004)$. When examining serum infliximab levels, 63 had sub-therapeutic levels of infliximab. 25 of $29(83 \%)$ who then underwent dose escalation had a clinical response. Six patients with sub-therapeutic levels changed to another anti-TNF, with two (33\%) responding $(\mathrm{p}<0.016)$. For both ATIs and drug level monitoring, this study provides some of the first evidence supporting the concept of applying this information in an algorithm such as Figure 1, with subsequently improved response rates. However, there are some limitations, including the retrospective acquisition of data and the definition of clinical response in this study.

Another retrospective review yielded conflicting results. Pariente and colleagues examined a cohort of 76 individuals with IBD who had ATIs and trough infliximab levels using ELISA and assessed for evidence of dose escalation ${ }^{36}$. In this cohort, 16 of 76 patients $(22.4 \%)$ 
developed detectable ATIs. Ten ATI-positive patients had an intensification of infliximab therapy and six $(60 \%)$ demonstrated a clinical response. These are considerably higher response rates than those appreciated by Afif and colleagues. Furthermore, the authors did not appreciate a significant association with trough levels of infliximab and response to dose intensification. However, one should interpret these results cautiously given the relatively small numbers of individuals, particularly regarding ATIs.

A recently published study by Vande Casteele and colleagues assessed the impact of ATIs and trough infliximab levels using both the standard ELISA assay and an HMSA-based assay in 90 patients with IBD $^{14}$. Fifty-three of 90 patients were discovered to be ATIpositive. However, when re-assessing the ATI status of these patients over multiple visits using the HMSA assay, 15 of these patients were noted to have transient antibodies. Of particular interest were the therapeutic implications of transient ATIs compared to sustained ATIs when dose escalation was attempted. In those with sustained ATIs, 25 of 38 underwent dose escalation, with only 4/25 (16\%) having a response. However, in those with transient ATIs, $69 \%$ had a clinical response ( $\mathrm{p}=0.0028$ ). The authors also appreciated that successful dose modification was associated with an increase in trough levels of infliximab. Therefore, in this study, the commonly suggested algorithm was supported. In addition, a unique population with transient ATIs was identified, in part due to the use of a novel new HMSA assay. Further study is needed, however, before widespread application of such new techniques can be recommended.

In addition to these retrospective studies, there is now also some prospective data assessing therapeutic drug monitoring. Vande Casteele and colleagues recently published an abstract examining the impact of prospective, long-term follow-up and therapeutic drug level monitoring. In this study, 123 patients with either $\mathrm{UC}$ or $\mathrm{CD}$ were randomized to dose intensification based on clinical response, and an additional 128 patients were randomized to dose modification based on drug levels, with one year of follow-up. Ultimately, $69 \%$ of those in the clinically-managed arm and $72 \%$ of those in the drug level-based arm were in clinical remission at 1 year $(\mathrm{P}=0.7)$. However, a significantly higher proportion of individuals fell within the pre-determined, ideal therapeutic range in the arm managed by levels ( $78 \%$ vs $56 \%, \mathrm{p}<0.001)$. ATIs were more common in the clinically-managed group, but overall rates were very low in this study. 37,38

There are also data supporting therapeutic drug monitoring as being more cost-effective. Steenholdt and colleagues performed a randomized, controlled trial of 69 individuals with secondary failure of infliximab. Of these, 36 patients underwent blind dose escalation of infliximab, and 33 were managed by ATI and infliximab levels. Response rates were similar between the two groups at 12 weeks, though costs were reduced in the ATI and trough levelguided therapy $(\mathrm{p}<0.001)$. As with the previously noted retrospective studies, the overall population size was relatively small. It is intriguing that even if there is not a significant clinical benefit, there may still be a subsequent economic benefit of guiding therapy by ATI and drug level status ${ }^{39}$. 


\section{Conclusions}

In summary, anti-TNF agents have had a significant impact in the treatment of IBD.

However, a significant number of patients lose response to these medications over time.

There is growing evidence that suggests that anti-TNF antibodies and anti-TNF drug levels may play a significant role in such clinical changes for a subset of patients; ATIs appear to be associated with shorter response duration and loss of response, particularly in nonscheduled dosing. Antibodies to infliximab are also associated with an increased risk of transfusion reaction. Finally, lower drug levels appear to be associated with a lack of response, particularly with infliximab. There is currently limited research on the impact of using this data to guide changes in therapy, though two retrospective studies and one prospective study appear to support the use of these tests in patients who have lost response before making changes to therapy. Lastly, there are some limitations with the assays used in prior studies, and the advent of newer assays may allow for the detection of new phenomena such as transient antibody formation. Given these factors, the utilization of antidrug antibody levels and anti-TNF serum trough levels is expanding.

\section{References}

1. Colombel JF, Sandborn WJ, Reinisch W, et al. Infliximab, azathioprine, or combination therapy for Crohn's disease. N Engl J Med. Apr 15; 2010 362(15):1383-1395. [PubMed: 20393175]

2. Lemann M, Mary JY, Duclos B, et al. Infliximab plus azathioprine for steroid-dependent Crohn's disease patients: a randomized placebo-controlled trial. Gastroenterology. Apr; 2006 130(4):10541061. [PubMed: 16618399]

3. D'Haens G, Baert F, van Assche G, et al. Early combined immunosuppression or conventional management in patients with newly diagnosed Crohn's disease: an open randomised trial. Lancet. Feb 23; 2008 371(9613):660-667. [PubMed: 18295023]

4. Sandborn WJ, Hanauer SB, Rutgeerts P, et al. Adalimumab for maintenance treatment of Crohn's disease: results of the CLASSIC II trial. Gut. Sep; 2007 56(9):1232-1239. [PubMed: 17299059]

5. Hanauer SB, Feagan BG, Lichtenstein GR, et al. Maintenance infliximab for Crohn's disease: the ACCENT I randomised trial. Lancet. May 4; 2002 359(9317):1541-1549. [PubMed: 12047962]

6. Schreiber S, Khaliq-Kareemi M, Lawrance IC, et al. Maintenance therapy with certolizumab pegol for Crohn's disease. The New England journal of medicine. Jul 19; 2007 357(3):239-250.

[PubMed: 17634459]

7. Cassinotti A, Travis S. Incidence and clinical significance of immunogenicity to infliximab in Crohn's disease: a critical systematic review. Inflammatory bowel diseases. Aug; 2009 15(8):12641275. [PubMed: 19235918]

8. Lichtenstein GR. Comprehensive review: antitumor necrosis factor agents in inflammatory bowel disease and factors implicated in treatment response. Therapeutic advances in gastroenterology. Jul; 2013 6(4):269-293. [PubMed: 23814608]

9. Imaeda H, Takahashi K, Fujimoto T, et al. Clinical utility of newly developed immunoassays for serum concentrations of adalimumab and anti-adalimumab antibodies in patients with Crohn's disease. Journal of gastroenterology. Apr 11.2013

10. Hanauer SB, Sandborn WJ, Rutgeerts P, et al. Human anti-tumor necrosis factor monoclonal antibody (adalimumab) in Crohn's disease: the CLASSIC-I trial. Gastroenterology. Feb; 2006 130(2):323-333. quiz 591. [PubMed: 16472588]

11. Sandborn WJ, Feagan BG, Stoinov S, et al. Certolizumab pegol for the treatment of Crohn's disease. N Engl J Med. Jul 19; 2007 357(3):228-238. [PubMed: 17634458]

12. Ben-Horin S, Yavzori M, Katz L, et al. The immunogenic part of infliximab is the $F\left(\mathrm{ab}^{\prime}\right) 2$, but measuring antibodies to the intact infliximab molecule is more clinically useful. Gut. Jan; 2011 60(1):41-48. [PubMed: 20519742] 
13. Ordas I, Mould DR, Feagan BG, Sandborn WJ. Anti-TNF monoclonal antibodies in inflammatory bowel disease: pharmacokinetics-based dosing paradigms. Clinical pharmacology and therapeutics. Apr; 2012 91(4):635-646. [PubMed: 22357456]

14. Vande Casteele N, Gils A, Singh S, et al. Antibody response to infliximab and its impact on pharmacokinetics can be transient. The American journal of gastroenterology. Jun; 2013 108(6): 962-971. [PubMed: 23419382]

15. Seow CH, Newman A, Irwin SP, Steinhart AH, Silverberg MS, Greenberg GR. Trough serum infliximab: a predictive factor of clinical outcome for infliximab treatment in acute ulcerative colitis. Gut. Jan; 2010 59(1):49-54. [PubMed: 19651627]

16. Khanna R, Sattin BD, Afif W, et al. Review article: a clinician's guide for therapeutic drug monitoring of infliximab in inflammatory bowel disease. Alimentary pharmacology \& therapeutics. Sep; 2013 38(5):447-459. [PubMed: 23848220]

17. Ainsworth MA, Bendtzen K, Brynskov J. Tumor necrosis factor-alpha binding capacity and antiinfliximab antibodies measured by fluid-phase radioimmunoassays as predictors of clinical efficacy of infliximab in Crohn's disease. The American journal of gastroenterology. Apr; 2008 103(4):944-948. [PubMed: 18028512]

18. Wang SL, Ohrmund L, Hauenstein S, et al. Development and validation of a homogeneous mobility shift assay for the measurement of infliximab and antibodies-to-infliximab levels in patient serum. Journal of immunological methods. Aug 31; 2012 382(1-2):177-188. [PubMed: 22691619]

19. Infliximab Concentration and Anti-Infliximab Antibody. LabHorizons: a Newsletter for Clients. Nov.2012 13(11-12):3.

20. Baert F, Noman M, Vermeire S, et al. Influence of immunogenicity on the long-term efficacy of infliximab in Crohn's disease. The New England journal of medicine. Feb 13; 2003 348(7):601608. [PubMed: 12584368]

21. Farrell RJ, Alsahli M, Jeen YT, Falchuk KR, Peppercorn MA, Michetti P. Intravenous hydrocortisone premedication reduces antibodies to infliximab in Crohn's disease: a randomized controlled trial. Gastroenterology. Apr; 2003 124(4):917-924. [PubMed: 12671888]

22. Vermeire S, Noman M, Van Assche G, Baert F, D'Haens G, Rutgeerts P. Effectiveness of concomitant immunosuppressive therapy in suppressing the formation of antibodies to infliximab in Crohn's disease. Gut. Sep; 2007 56(9):1226-1231. [PubMed: 17229796]

23. Hanauer SB, Wagner CL, Bala M, et al. Incidence and importance of antibody responses to infliximab after maintenance or episodic treatment in Crohn's disease. Clinical gastroenterology and hepatology: the official clinical practice journal of the American Gastroenterological Association. Jul; 2004 2(7):542-553. [PubMed: 15224278]

24. Maser EA, Villela R, Silverberg MS, Greenberg GR. Association of trough serum infliximab to clinical outcome after scheduled maintenance treatment for Crohn's disease. Clinical gastroenterology and hepatology: the official clinical practice journal of the American Gastroenterological Association. Oct; 2006 4(10):1248-1254. [PubMed: 16931170]

25. Rutgeerts P, Sandborn WJ, Feagan BG, et al. Infliximab for induction and maintenance therapy for ulcerative colitis. The New England journal of medicine. Dec 8; 2005 353(23):2462-2476. [PubMed: 16339095]

26. Van Assche G, Magdelaine-Beuzelin C, D'Haens G, et al. Withdrawal of immunosuppression in Crohn's disease treated with scheduled infliximab maintenance: a randomized trial. Gastroenterology. Jun; 2008 134(7):1861-1868. [PubMed: 18440315]

27. Sandborn WJ, van Assche G, Reinisch W, et al. Adalimumab induces and maintains clinical remission in patients with moderate-to-severe ulcerative colitis. Gastroenterology. Feb; 2012 142(2):257-265. e251-253. [PubMed: 22062358]

28. Karmiris K, Paintaud G, Noman M, et al. Influence of trough serum levels and immunogenicity on long-term outcome of adalimumab therapy in Crohn's disease. Gastroenterology. Nov; 2009 137(5):1628-1640. [PubMed: 19664627]

29. Sandborn WJ, Abreu MT, D'Haens G, et al. Certolizumab pegol in patients with moderate to severe Crohn's disease and secondary failure to infliximab. Clin Gastroenterol Hepatol. Aug; 2010 8(8):688-695. e682. [PubMed: 20451663] 
30. Keizer RJ, Huitema AD, Schellens JH, Beijnen JH. Clinical pharmacokinetics of therapeutic monoclonal antibodies. Clinical pharmacokinetics. Aug; 2010 49(8):493-507. [PubMed: 20608753]

31. Reinisch W, Feagan BG, Rutgeerts PJ, et al. Infliximab Concentration and Clinical Outcome in Patients With Ulcerative Colitis. Gastroenterology. 2012; 142(5):S114. [Abstract].

32. Li J, Paulson SK, Chiu Y-L, Robinson Anne, Lomax KG, Pollac PF. Evaluation of Potential Correlations Between Serum Adalimumab Concentration and Remission in Patients With Crohn's Disease in Classic I and II. Gastroenterology. 2010; 138(5):S101. [Abstract].

33. Sandborn WJ, Schreiber S, Hanauer SB, Colombel JF, Bloomfield R, Lichtenstein GR. Reinduction with certolizumab pegol in patients with relapsed Crohn's disease: results from the PRECiSE 4 Study. Clinical gastroenterology and hepatology: the official clinical practice journal of the American Gastroenterological Association. Aug; 2010 8(8):696-702. e691. [PubMed: 20363366]

34. Sandborn WJ, Hanauer SB, Pierre-Louis B, Lichtenstein GR. Certolizumab Pegol Plasma Concentration and Clinical Remission in Crohn's Disease. Gastroenterology. 2012; 142(5):S563. [Abstract Su2079].

35. Colombel JF, Feagan BG, Sandborn WJ, Van Assche G, Robinson AM. Therapeutic drug monitoring of biologics for inflammatory bowel disease. Inflammatory bowel diseases. Feb; 2012 18(2):349-358. [PubMed: 22021134]

36. Pariente B, Pineton de Chambrun G, Krzysiek R, et al. Trough levels and antibodies to infliximab may not predict response to intensification of infliximab therapy in patients with inflammatory bowel disease. Inflammatory bowel diseases. Jul; 2012 18(7):1199-1206. [PubMed: 22127789]

37. Vande Casteele N, Compernolle G, Ballet V, et al. Results on the Optimisation Phase of the Prospective Controlled Trough Level Adapted Infliximab Treatment (TAXIT) Trial. Gastroenterology 2012. 2012; 142(5):S211-S212.

38. Vande Casteele N, Gils A, Ballet V, et al. Randomised Controlled Trial of Drug Level verus Clinically Based Dosing of Infliximan Maintenance Therapy in IBD: Final Results of the TAXIT study. United European Gastroenterology Journal. Oct.2013 1:A1. 2013.

39. Steenholdt C, Brynskov J, Thomsen OO, et al. Individualised therapy is more cost-effective than dose intensification in patients with Crohn's disease who lose response to anti-TNF treatment: a randomised, controlled trial. Gut. Jul 22.2013

\section{Biography}

Gary R. Lichtenstein has received consultancy payments from Abbott Corporation/Abbvie, Alaven, Bristol-Myers Squibb, Elan, Ferring, Hospira, Janssen Orthobiotech, Millenium Pharmaceuticals, Ono Pharmaceuticals, Pfizer Pharmaceuticals, Prometheus Laboratories, Inc., Salix Pharmaceuticals, Santarus, Shire Pharmaceuticals, Takeda, UCB, and Warner; honoraria from Ironwood and Luitpold/American Regent; and a research grant from Warner Chilcotte. 


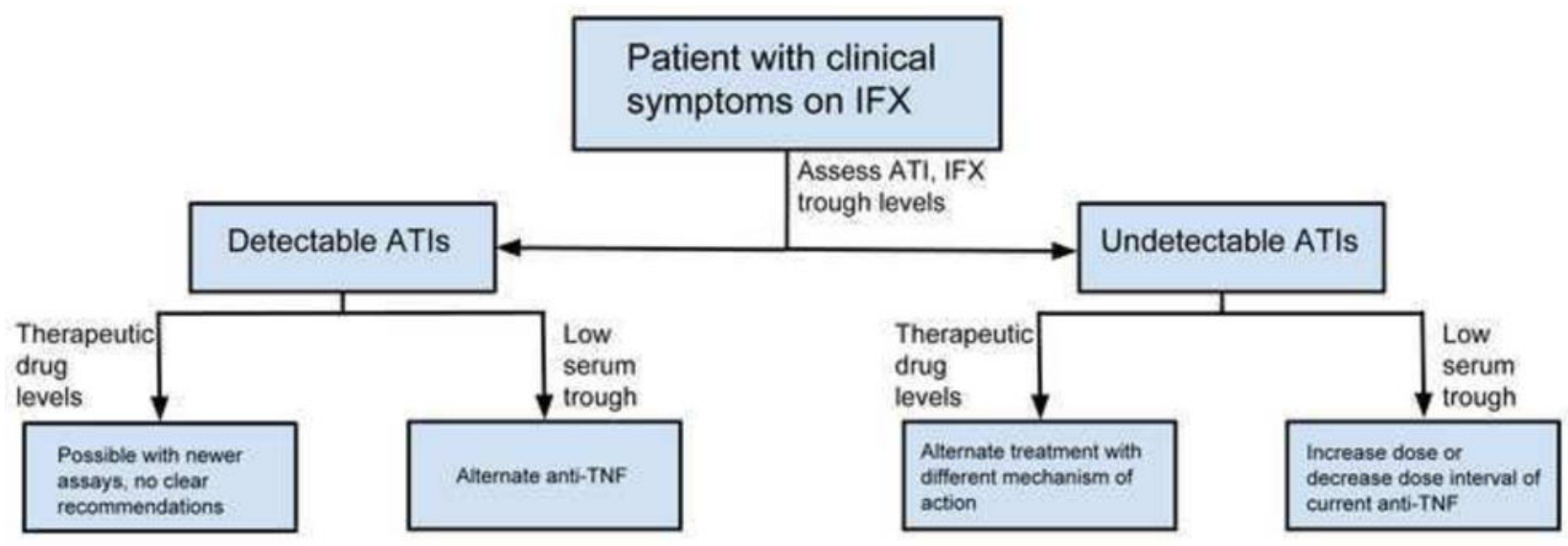

Figure 1. 


\section{Table 1}

Assays used to detect antibodies to infliximab

\begin{tabular}{|c|c|c|}
\hline Assay type & Advantages & Disadvantages \\
\hline ELISA & - $\quad$ Ease of administration & $\begin{array}{ll}\text { - } & \text { False-positives } \\
\text { - } & \text { Interference by anti-TNF }\end{array}$ \\
\hline RIA & $\begin{array}{l}\text { - } \quad \text { Can detect ATIs in presence of infliximab } \\
\text { - } \quad \text { Can measure infliximab levels } \\
\text { More resistant to cross-reactivity with other } \\
\text { antibodies }\end{array}$ & $\begin{array}{l}\text { - } \\
\text { Pequires use of radioactive isotopes } \\
\text { Prolonged incubation time for } \\
\text { equilibration of binding }\end{array}$ \\
\hline HMSA & $\begin{array}{l}\text { - } \begin{array}{l}\text { Not sensitive to interference by other } \\
\text { antibodies }\end{array} \\
\text { - } \quad \text { Increased sensitivity compared to ELISA } \\
\text { - } \begin{array}{l}\text { Able to measure all subtypes of } \\
\text { immunoglobulins to infliximab }\end{array} \\
\text { - } \quad \text { No radioactive component as in RIA }\end{array}$ & $\begin{array}{l}\text { - } \quad \text { Requires further validation, relatively } \\
\text { new assay }\end{array}$ \\
\hline Electrochemiluminescence & $\begin{array}{l}\text { - } \quad \text { Can measure ATIs in the presence of } \\
\text { infliximab } \\
\text { - } \quad \text { Standardized lab equipment } \\
\text { - } \quad \text { No radioactive components }\end{array}$ & $\begin{array}{l}\text { - } \quad \text { ATIs interfere with drug level detection } \\
\text { Not yet validated for use with } \\
\text { infliximab, relatively new assay }\end{array}$ \\
\hline
\end{tabular}




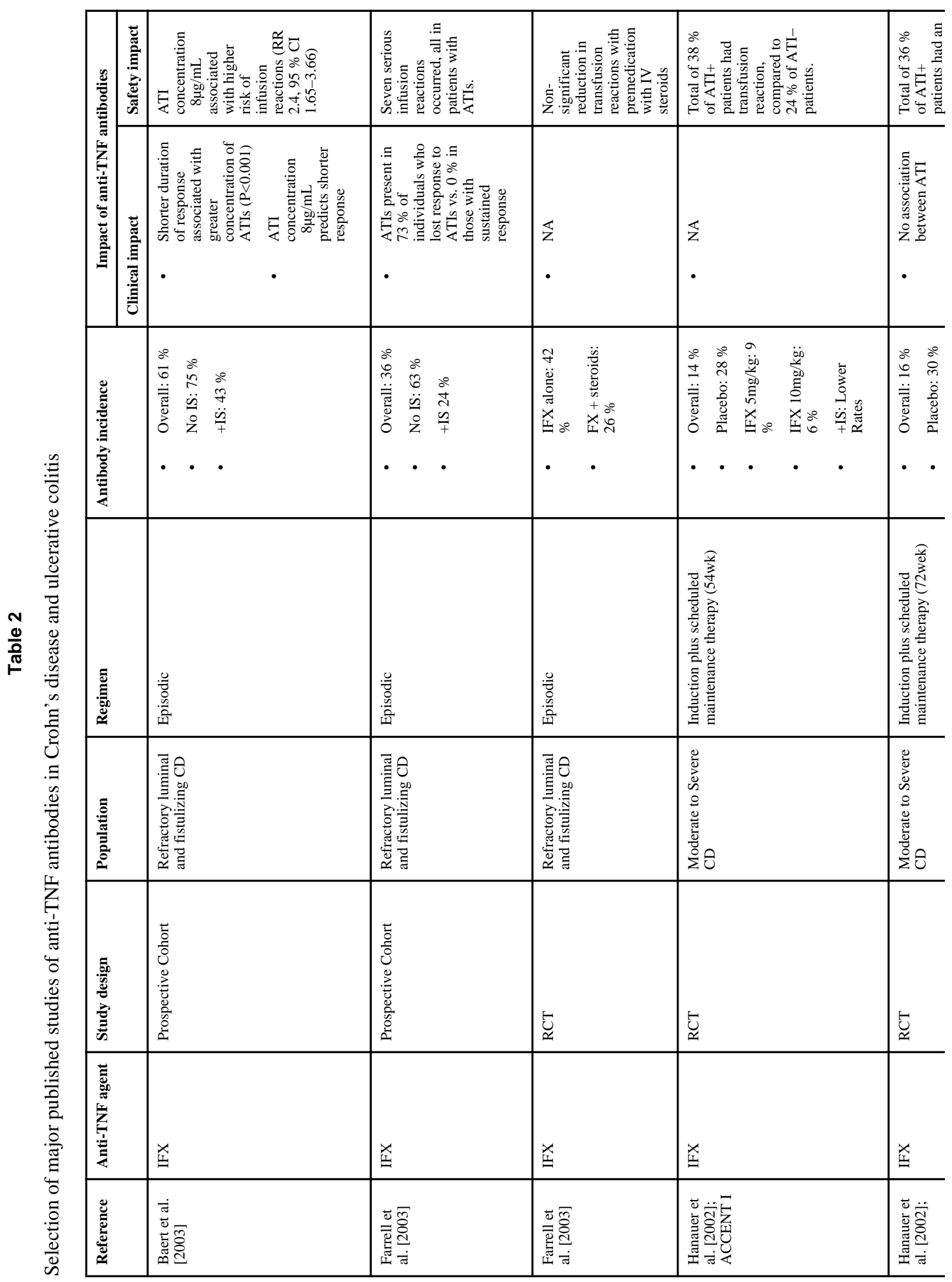

Curr Treat Options Gastroenterol. Author manuscript; available in PMC 2015 March 01. 


\begin{tabular}{|c|c|c|c|c|c|c|}
\hline 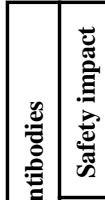 & 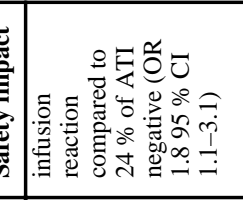 & 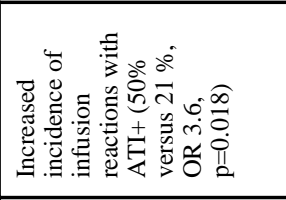 & 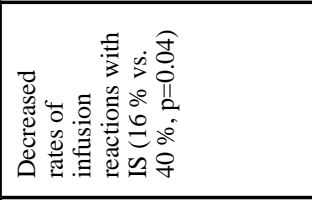 & $\mathbb{z}$ & $\underline{z}$ & 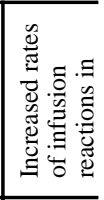 \\
\hline 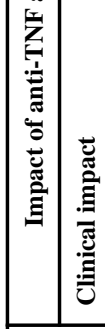 & 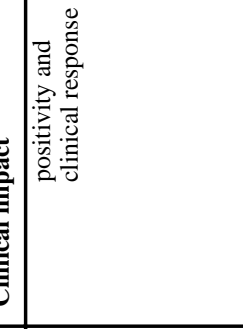 & 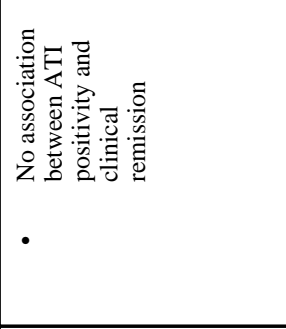 & 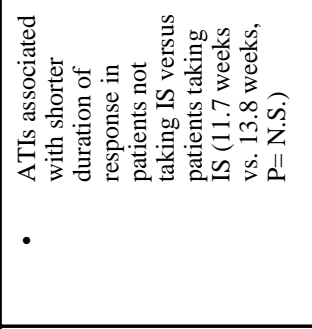 & 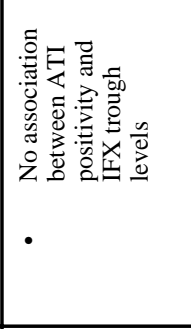 & 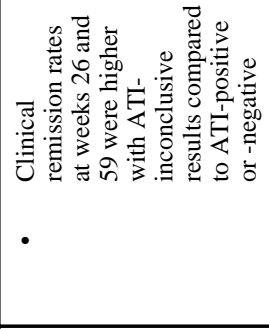 & 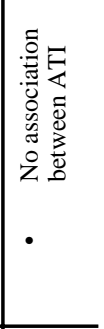 \\
\hline 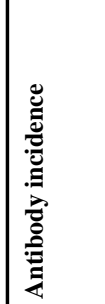 & 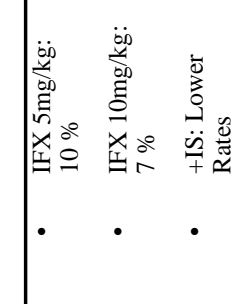 & 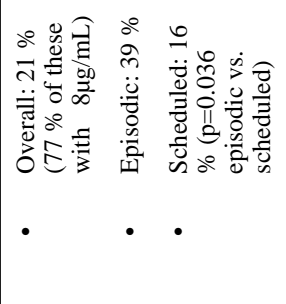 & 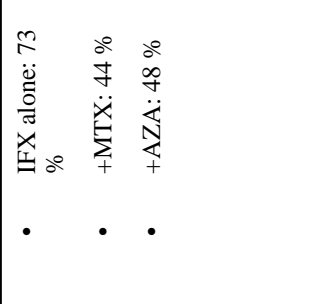 & 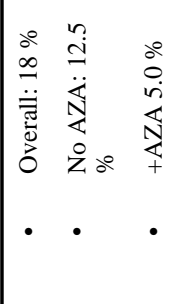 & 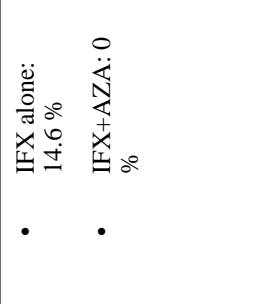 & 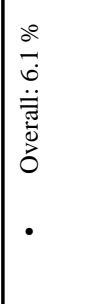 \\
\hline 童 & & 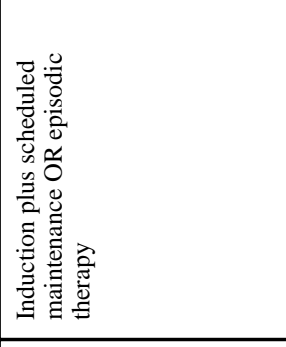 & $\begin{array}{l}\text { 竞 } \\
\text { : } \\
\text { 童 }\end{array}$ & 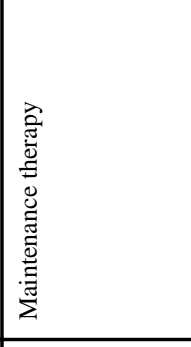 & 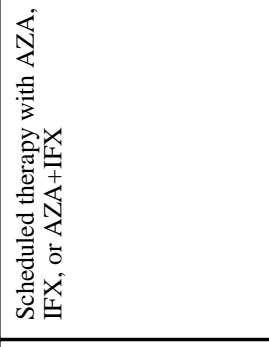 & 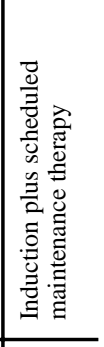 \\
\hline 言 & & 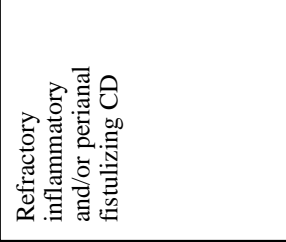 & 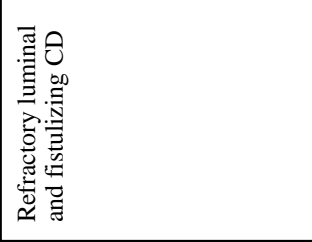 & 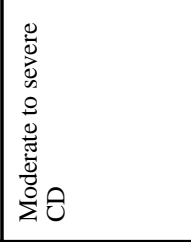 & 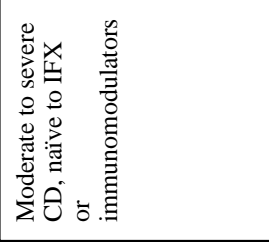 & 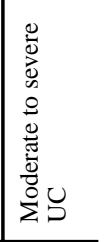 \\
\hline 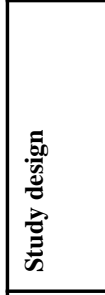 & & 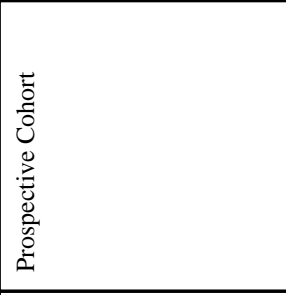 & 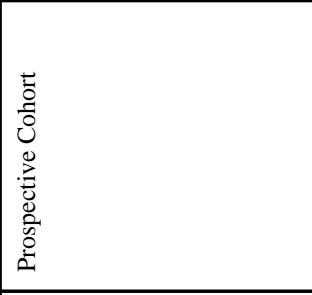 & 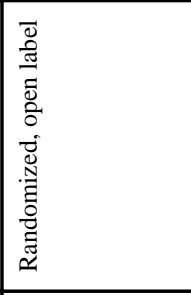 & $\tilde{\varkappa}^{-}$ & $E_{\dddot{\varkappa}}$ \\
\hline 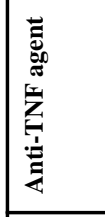 & & 希 & 屆 & 厓 & 厓 & 宏 \\
\hline 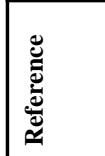 & 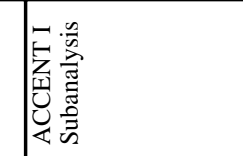 & 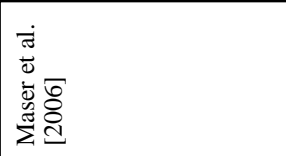 & 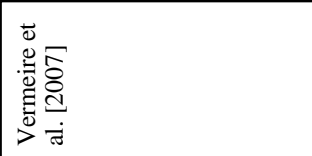 & 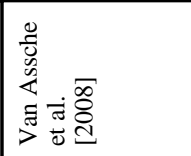 & 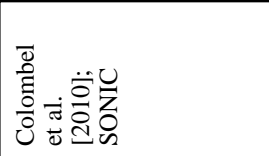 & 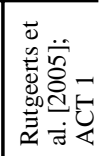 \\
\hline
\end{tabular}




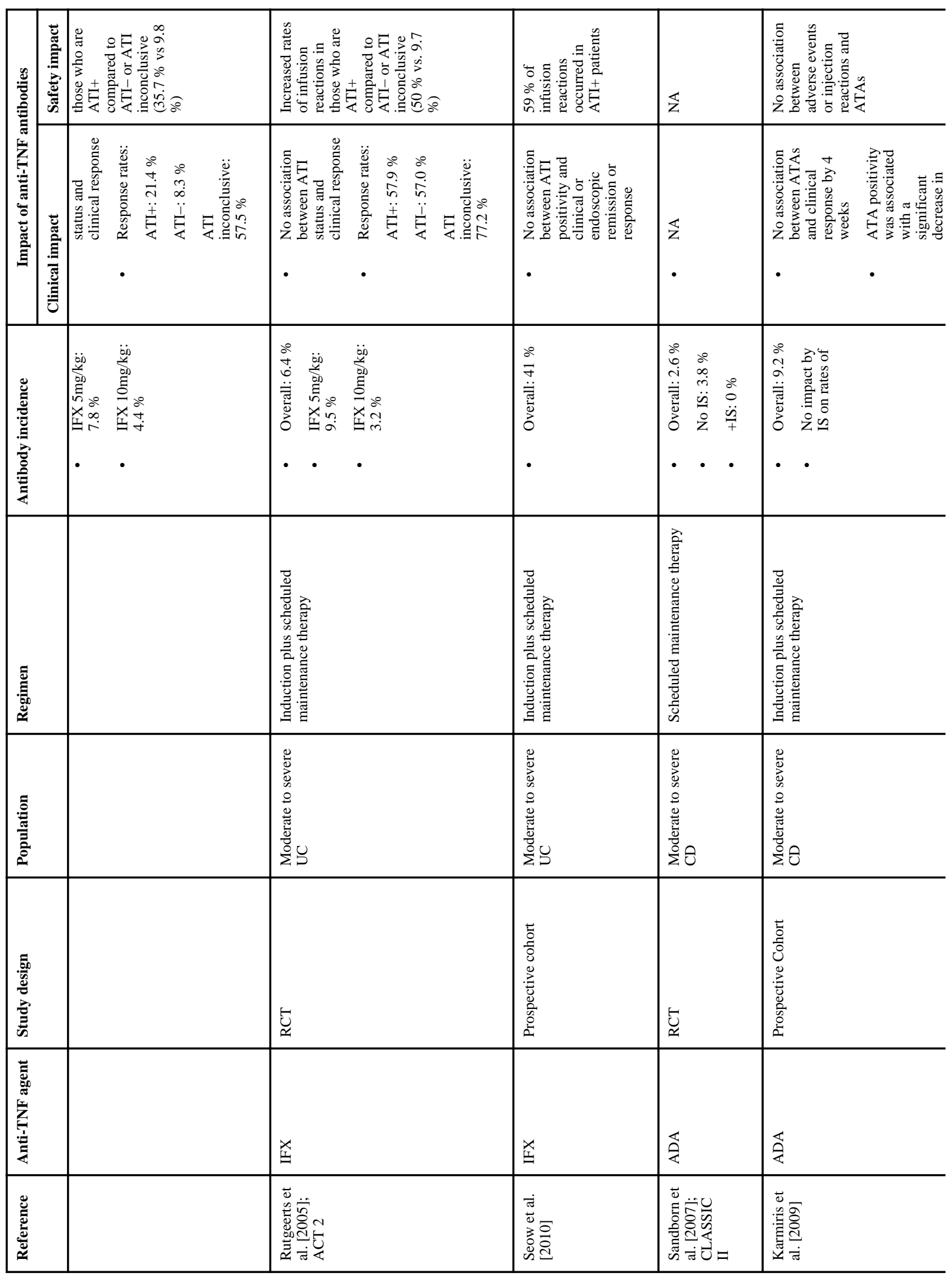




\begin{tabular}{|c|c|c|c|}
\hline 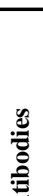 & 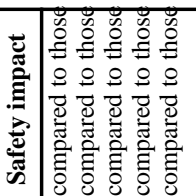 & $\overleftrightarrow{z}$ & $\overleftrightarrow{z}$ \\
\hline 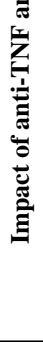 & 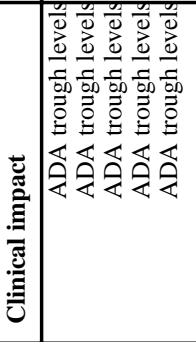 & 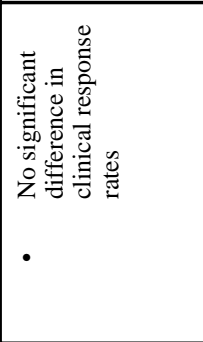 & 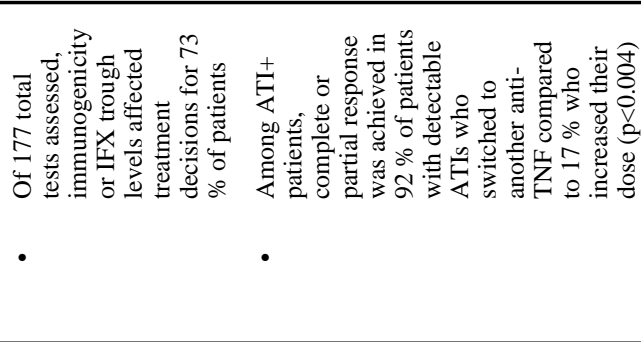 \\
\hline 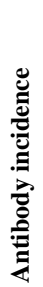 & & 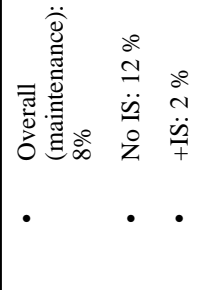 & 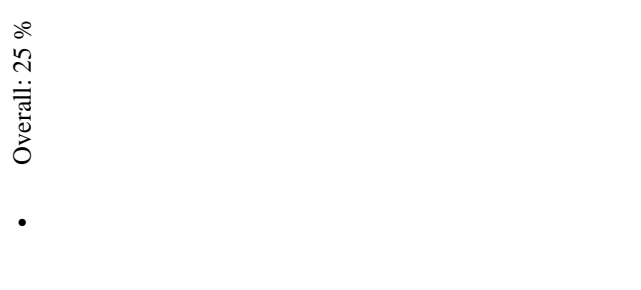 \\
\hline 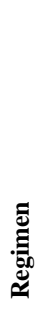 & & 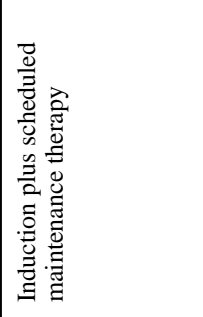 & \\
\hline 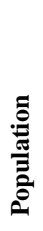 & & 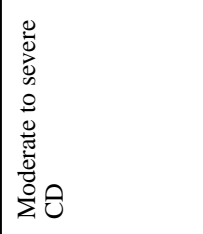 & 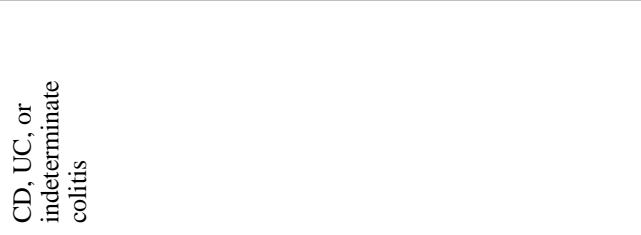 \\
\hline 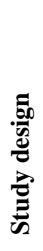 & & 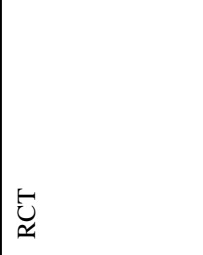 & 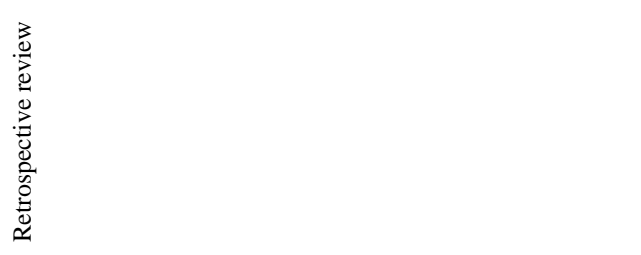 \\
\hline 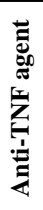 & & $\hat{U}$ & 在 \\
\hline 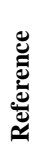 & & 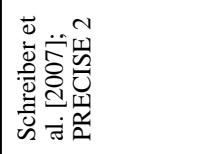 & 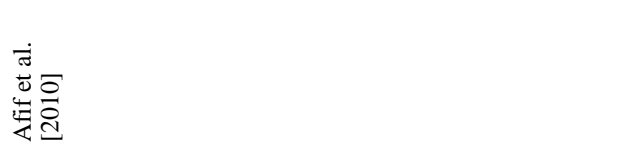 \\
\hline
\end{tabular}

